\title{
Mapping the Interoperability Landscape for Networked Information Retrieval
}

\author{
William E. Moen \\ School of Library and Information Sciences, Texas Center for Digital Knowledge, \\ University of North Texas, P.O. Box 311068, Denton, Texas 76203 \\ 940-565-3563 \\ wemoen@unt.edu
}

\begin{abstract}
Interoperability is a fundamental challenge for networked information discovery and retrieval. Often treated monolithically in the literature, interoperability is multifaceted and can be analyzed into different types and levels. This paper discusses an approach to map the interoperability landscape for networked information retrieval as part of an interoperability assessment research project.
\end{abstract}

\section{Categories and Subject Descriptors}

H.3.7 [Information Storage and Retrieval]: Digital Libraries standards, systems issues, user issues.

\section{General Terms}

Standardization

\section{Keywords}

Interoperability; networked information discovery and retrieval; Z39.50, testbeds.

\section{INTRODUCTION}

The ability of two information systems to communicate, execute instructions, share data, or otherwise interact is a fundamental requirement in the networked environment. Typically these interactions are subsumed under the term interoperability. In the traditional library automation environment and more recently in the digital library context, we have recognized the complexity of linking information retrieval systems. Yet, there are increased expectations for seamless, transparent, and reliable access and sharing of information in and between digital libraries. Increasingly the literature identifies interoperability as one of the fundamental problem facing networked information discovery and retrieval (NIDR) [3].

This paper outlines a preliminary and evolving framework for addressing interoperability. Mapping the NIDR interoperability landscape is part of a broader research and demonstration project

Permission to make digital or hard copies of all or part of this work for personal or classroom use is granted without fee provided that copies are not made or distributed for profit or commercial advantage and that copies bear this notice and the full citation on the first page. To copy otherwise, or republish, to post on servers or to redistribute to lists, requires prior specific permission and/or a fee.

$J C D L ' 01$, June 24-28, 2001, Roanoke, Virginia, USA.

Copyright 2001 ACM 1-58113-345-6/01/0006...\$5.00. for assessing interoperability. The U.S. federal Institute of Museum and Library Services awarded a National Leadership Grant to the School of Library and Information Sciences and Texas Center for Digital Knowledge at the University of North Texas to establish a research and demonstration Z39.50 interoperability testbed [5].

A map or conceptual framework of interoperability allows us to situate our focal activities since not all types of interoperability will be assessed through the initial testbed. By identifying the multiple factors that threaten interoperability, we can control some of those factors in the testbed while acknowledging that subsequent phases of the research can address other factors. For example, the initial Z39.50 interoperability testbed focuses on three types or levels of interoperability: protocol syntax level, protocol service level, and semantic level. Each of these levels, and particularly the semantic level, is multifaceted. Lynch and Garcia-Molina describe semantic interoperability as a "grand challenge" research problem [3]. The testbed focuses on some aspects of semantic interoperability.

Similar to Paepcke, et al. [1], we suggest that multiple levels and categories of interoperability can be identified, and these may differ depending on the application. Too often the literature treats interoperability monolithically or simply from a system perspective (i.e., the level of two systems interacting). Mapping the interoperability landscape can help focus attention on specific interoperability problems and assessment methodologies. We suggest further that the degree of interoperability between information systems may be dependent on the distance between communities whose information systems attempt to interact. Further, it is ultimately the user who benefits when systems interoperate, and we propose that user assessments of interoperability should be factored into the methodology.

\section{DEFINITIONS}

In the context of the networked environment, a number of definitions of interoperability surface $[2,6]$. Miller goes beyond a system orientation and describes a more expansive perspective on interoperability. He suggests multiple aspects of the concept including Technical, Semantic, Inter-community, and Legal [4].

Within the context of digital libraries and networked information retrieval, we assume the following operating principle: systems will interoperate. Miller's approach, however, underscores the principle that not only systems but also organizations will need to interoperate. This brings attention to various environmental factors that can affect interoperability. 


\section{INTEROPERABILITY FACTORS}

A number of diverse factors challenge interoperability:

- Multiple and disparate operating and IR systems

- Multiple protocols

- Multiple metadata schemes

- Multiple data formats

- Multiple languages and character sets

- Multiple vocabularies, ontologies, and disciplines.

Our map of interoperability will account for a variety of factors.

\section{COMMUNITY AND DOMAIN CONTEXTS FOR INTEROPERABILITY}

The context of "information communities" provides a way to frame the challenges of achieving interoperability. The diversity of factors above may be reduced within a particular community.

For cross-catalog information retrieval in traditional libraries, the diversity listed above is radically reduced. Data in the catalogs are relatively homogenous, and there is commonality in the metadata scheme for structuring the records, etc. The challenges to achieve interoperability in a virtual catalog application may be less than in cases when one crosses community boundaries. For example, if a user queries repositories of library bibliographic records and a museum's object records concurrently through Z39.50, diversity of metadata schemes and vocabulary increases.

Our preliminary map proposes the following NIDR communities:

- Focal-community NIDR: factors affecting interoperability are minimized (e.g., libraries)

- Extended-community NIDR: increased diversity (e.g., cultural heritage information held by libraries and museum)

- Extra-community NIDR: factors affecting interoperability are maximized (e.g., libraries interacting with geospatial repositories).

Challenges to interoperability NIDR increase (and likely the costs of achieving interoperability increase) as one moves further outside of a focal community. Figure 1 illustrates potential interaction among communities.

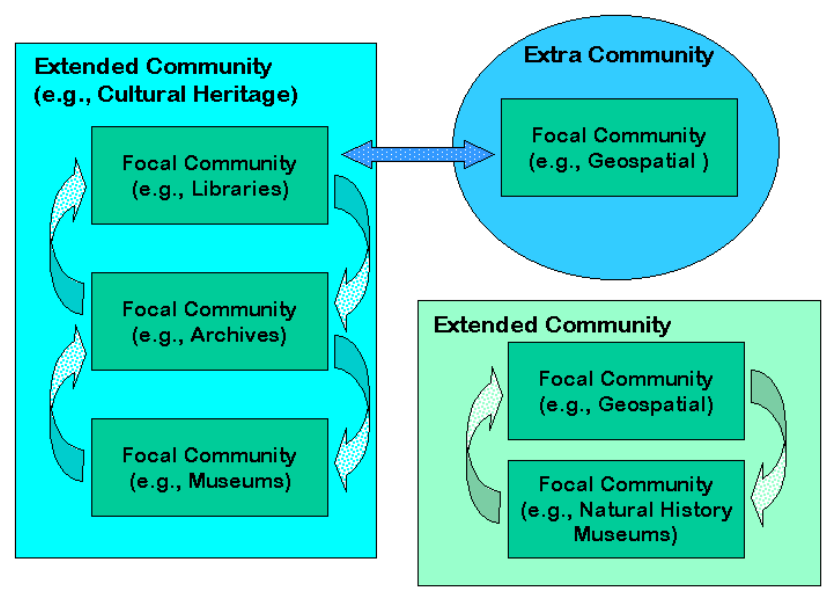

Figure 1. Information Retrieval Among/Across Communities
Another useful perspective for the interoperability conceptual map is in terms of domains. There may be some overlap here with the perspective of communities, but addressing differences in domains can highlight factors such as vocabularies and ontologies. Our mapping identifies the following two categories:

- Intra-domain/discipline

- Extra-domain/discipline.

As in the case of communities, the challenges to interoperability within a domain may be less than between domains. Semantic differences may present major barriers to interoperability.

Within a community or domain, relative homogeneity reduces interoperability challenges. Heterogeneity increases as one moves outside of a focal community/domain, and interoperability is likely more costly and more difficult to achieve.

\section{SUMMARY}

The work on mapping the interoperability landscape is in its initial phase. The resulting map situates our interoperability assessment research in that landscape and provides points of reference to other researchers for assessing and overcoming interoperability problems. Ultimately, this map may assist in realistically assessing the challenges and costs in making real the promises of providing seamless and transparent access (i.e., interoperability) within the networked environment.

\section{ACKNOWLEDGMENTS}

We thank the U.S. federal Institute of Museum and Library Services for support of the interoperability testbed and papers resulting from our research.

\section{REFERENCES}

[1] Paepcke, A., et al. Interoperability for digital libraries worldwide. Communications of the ACM 41(April 1998), $33-43$.

[2] Lynch, C. Interoperability: The standards challenge for the 1990s. Wilson Library Bulletin, 67 (March 1993), 38-42.

[3] Lynch, C. \& Garcia-Molina, H. Interoperability, scaling, and the digital libraries research agenda: A report on the May 1819, 1995 IITA digital libraries workshop. (1995). Available URL:

http:/www-diglib.stanford.edu/diglib/pub/reports/iita-dlw/

[4] Miller, P. Interoperability: What it is and why should I want it. Ariadne, 24. Available URL:

http://www.ariadne.ac.uk/issue24/interoperability/intro.html

[5] Moen, W. E. Realizing the vision of networked access to library resources: An applied research and demonstration project to establish and operate a Z39.50 interoperability testbed. (February 2000). Available URL: http://www.unt.edu/zinterop

[6] Preston, C.M. \& Lynch, C.A. Interoperability and conformance issues in the development and implementation of the government information locator service (GILS). In W.E. Moen and C.R. McClure, The Government Information Locator Service (GILS). Syracuse, NY: School of Information Studies, Syracuse University (1994). 